Abstract ID: 34

\title{
Epigenetic methylation status of P16, MGMT and SPOCK2 in diffuse Large B cell lymphoma
}

\author{
Lailatul Jalilah $^{\mathrm{a}}$ | Aziah Ismail ${ }^{\mathrm{b}} \mid$ Norlelawati A. Talib $^{\mathrm{c}}$ | Naznin Muhammad ${ }^{\mathrm{c}}$ | Faezahtul Arbaeyah \\ Hussain $^{\text {I }}$ Norafiza Zainuddin ${ }^{\mathrm{a}}$ \\ ${ }^{a}$ Department of Biomedical Science, Kulliyyah of Allied Health Sciences, International Islamic \\ University Malaysia \\ ${ }^{b}$ Institute for Research in Molecular Medicine (INFORMM), Universiti Sains Malaysia \\ 'Department of Pathology and Laboratory Medicine, Kulliyyah of Medicine, International Islamic \\ University Malaysia \\ ${ }^{d}$ Department of Pathology, School of Medical Sciences, Universiti Sains Malaysia
}

Introduction: Epigenetic methylation has been implicated in the pathogenesis of diffuse large B cell lymphoma (DLBCL). This study investigated the methylation status of $p 16$, MGMT and SPOCK2. Aberrantly methylated $p 16$ and MGMT have been linked to DLBCL, but not SPOCK2. p16 inhibits cyclin-dependent kinase, which results in retinoblastoma phosphorylation and blockage of cell cycle at G1 phase. MGMT removes alkyl adduct at $O^{6}$ guanine, thus preventing lethal cross-links. SPOCK2, an extracellular chondroitin and heparin sulfate proteoglycans, abolishes the inhibition of membrane-type 1-matrix metalloproteinase which might enhance the angiogenesis. The absence of SPOCK2 methylation was therefore hypothesized in the majority of cases in this study. Methods: Extracted DNA from 88 formalin-fixed paraffin-embedded (FFPE) tissues of DLBCL were subjected to bisulfite conversion followed by methylation-specific PCR (MSP) analysis for p16, MGMT and SPOCK2 methylation. p16 methylation was also quantified in 16 samples through pyrosequencing assay. Results: p16 methylation was observed in 65/88 (74\%) samples by MSP. Pyrosequencing detected $p 16$ methylation in all 16 samples ranging from $18 \%$ to $81 \%$. MGMT methylation was detected in all $88(100 \%)$ cases. Methylated SPOCK2 was found in $83(94.3 \%)$ samples. There was a significant association between $p 16$ methylation status with patients above 50 years of age $(p=0.04)$. Conclusions: These preliminary discoveries may serve as a good platform in order to gain a comprehensive overview on the epigenetics contribution in the pathogenesis of DLBCL. Pyrosequencing is a robust tool in detecting and quantifying methylation.

KEYWORDS: DLBCL, epigenetics, MSP, pyrosequencing 